\title{
Keck Interferometer science: present and future
}

\section{Rachel L. Akeson}

Rachel L. Akeson, "Keck Interferometer science: present and future," Proc. SPIE 5491, New Frontiers in Stellar Interferometry, (20 October 2004); doi: $10.1117 / 12.551592$

SPIE Event: SPIE Astronomical Telescopes + Instrumentation, 2004, Glasgow, United Kingdom 


\title{
Keck Interferometer Science: Present and Future
}

\author{
Rachel Akeson \\ Michelson Science Center \\ Pasadena CA 91125, USA \\ rla@ipac.caltech.edu
}

\section{Introduction}

The Keck Interferometer is a NASA funded project developed by the Jet Propulsion Laboratory, the William M. Keck Observatory and the Michelson Science Center at the California Institute of Technology. A technical description of the interferometer is given elsewhere in this volume ${ }^{1}$. This paper will discuss the science topics and goals of the Keck Interferometer project, including a brief description of the Key Science projects, the science projects executed to date and the current availability of the interferometer for new projects. The Keck Interferometer Project consists of the Keck-Keck Interferometer, which combines the two Keck 10-meter telescopes on an 85-meter baseline, and the Outrigger Telescopes Project, a proposal to add four to six 1.8-meter telescopes that would work in conjunction with the two Kecks.

\section{Observing modes and Key Science projects}

\subsection{Observing modes}

The Keck-Keck Interferometer (KI) combines the two $10 \mathrm{~m}$ Keck telescopes in the following modes:

- High sensitivity fringe visibility $\left(V^{2}\right)$ measurements. This mode combines the adaptive-optics (AO) corrected beams from the two Kecks for measurements of fringe amplitude in the near-infrared and is currently operational. Both telescopes are equipped with adaptive optics systems ${ }^{2}$, which are required for $K$-band interferometer observations. The interferometer field of view on the sky is $\sim 50$ milliarcseconds in the $K$ band, as set by a single-mode fiber ahead of the detector; the interferometer is not sensitive to emission outside of this field of view. The fringe tracker uses a 4 bin synchronous fringe demodulation algorithm ${ }^{3}$, similar to that used at the Palomar Testbed Interferometer ${ }^{4}$.

- Infrared nulling at $10 \mu \mathrm{m}$. Using a nulling beam combiner to suppress on-axis light this mode allows detection of faint sources of emission in the same field as bright sources, such as a dust cloud surrounding a central star. This mode uses a dedicated $10 \mu \mathrm{m}$ camera and more details are given elsewhere in this volume ${ }^{5}$.

- Differential-phase interferometry. Using a precision fringe detector and simultaneous multi-color observations of fringe position in the near infrared, this mode will be used to detect the fringe shift caused by sources with different near-infrared spectra contributing to the measured fringe. This mode uses a fringe tracking system with sensitivity in the $\mathrm{K}$ and $\mathrm{L}$ bands $(2.0$ to $3.9 \mu \mathrm{m})$. More details on this mode are given elsewhere in this volume ${ }^{6}$.

NASA has proposed to add four to six 1.8-m outrigger telescopes, providing a range of baseline lengths of 30-135 m. With the outrigger telescopes, two additional modes would be provided:

- High sensitivity imaging. With four outrigger telescopes and two Kecks, 15 baselines would be available for synthetic aperture imaging; nine of these baselines would include at least one Keck, providing excellent sensitivity. Cophasing of the array on a nearby bright star allows increased sensitivity, and the large collecting area of the Kecks increases the cophasing limiting magnitude, and hence sky coverage.

- Narrow-angle astrometry. The outrigger telescopes would provide long near-orthogonal baselines for an astrometric search for planets with a planned sensitivity of 30 microarcseconds per hour. Cophasing on the 
target star would be used to increase sensitivity for detection of an isoplanatic astrometric reference; operation over small fields with long baselines allows high accuracy through atmospheric turbulence.

\subsection{Key Science projects}

NASA has identified three Key Science projects, the first two of which utilize the collecting area of the 10-meter telescopes in the Keck-Keck Interferometer and the third of which would utilize the proposed Outrigger Telescopes.

1. Measurements of exo-zodiacal emission from stars using nulling interferometry: The goal of this project is to measure the level of zodiacal dust around potential targets for the Terrestrial Planet Finder mission (section 5) down to a level of 10 times the zodiacal emission in our own solar system.

2. Characterization of Hot Jupiters using differential phase: The goal of this project is to directly detect the signature of Hot Jupiters (exo-planets within $0.1 \mathrm{AU}$ of their parent star) using precise relative phase measurements to a level of 3 milliradians. These observations will allow determination of the true planet mass by measuring the orbit and low resolution spectral characterization across the $\mathrm{K}$ and $\mathrm{L}$ bands (section 6).

3. Astrometric observations of stars within 20 parsecs. Using narrow angle astrometry with a precision of 30 microarcseconds per hour, this project would search for planets down to Uranus-mass around stars out to 20 parsecs. This mode involves the use of the proposed Outrigger Telescope Array.

In addition to these specific key projects, all modes of the interferometer will be available for studies of general astrophysics.

\section{Current science status}

The Keck-Keck Interferometer obtained first fringes in March, 2001 and started science observations in the visibility amplitude mode in June, 2002. Four shared-risk teams were selected through a NASA research announcement issued in March, 2001 to assist the project in debugging the Keck-Keck Interferometer modes: visibility amplitude science, nulling and differential phase. The programs chosen are listed in section 3.1. In April 2004, the project passed an operational readiness review for the visibility amplitude mode.

Visibility amplitude observations with the interferometer are now operational and proposals are submitted through the normal Keck Observatory process. NASA will support observations from the Keck community up to $50 \%$ over the NASA usage. In the 2004B semester, that results in a maximum allocation of 6 interferometer nights from the Caltech, UC, NASA and UH time allocation committees. Information on the current sensitivity and observing parameters for the interferometer are available from the Michelson Science Center (MSC) at http://msc.caltech.edu/KISupport/index.html. In June 2004, the tracking limits are $\mathrm{R}$ band magnitude $\left(0.66\right.$ microns) $<10.5$ (as faint as $11^{\text {th }}$ magnitude for very red objects) and $\mathrm{K}$ band magnitude ( 2.2 microns) < 9.2. Proposal preparation support is also available from the MSC.

\subsection{Shared-risk science programs}

The shared-risk team PIs and proposal titles are:

- William Danchi, Goddard Space Flight Center, Multi-wavelength observations of circumstellar material

- Shrinivas Kulkarni, California Institute of Technology, Stellar astronomy with the Keck Interferometer

- John Monnier, University of Michigan, Resolving young stellar objects on sub-AU scales: The inner accretion disks of $\mathrm{T}$ Tauri and Herbig Ae/Be stars

- Wesley Traub, Harvard-Smithsonian Center for Astrophysics, Circumstellar disk detection with the Keck Nuller

\section{Visibility amplitude mode}

\subsection{Visibility amplitude data and interpretation}


Interferometer data with limited uv (spatial frequency) coverage, such as the single baseline of the Keck-Keck modes, can not be Fourier Transformed to provide an image of the source in the sky plane, but instead are compared to models of source emission. This technique is best applied to sources with relatively simple morphologies on the size scales relevant to the interferometer. Despite this limitation, interferometer measurements are currently the only way to probe size scales of a few milliarcseconds in the near-infrared and can therefore significantly improve our understanding of the physical properties of a wide range of astronomical objects.

At KI, observations consist of a series of interleaved integrations on the astronomical targets and calibrators. Each integration includes 120 seconds of fringe data followed by a background and foreground measurement. The balance of flux between the two arms is measured 5 times during the fringe tracking. The calculated science quantity is the visibility amplitude squared, which is an unbiased quantity, normalized such that an unresolved object has $\mathrm{V}^{2}=1.0$. The system visibility, the instrumental response to a point source, is measured with respect to the calibrator stars. Source and calibrator data are corrected for biases using sky calibrations as described by Ref. 4 and averaged into blocks of 5 seconds each. The data reduction and averaging to this level are done as part of the automated pipeline processing. Data are then calibrated for the system visibility?

Keck Interferoneter visibility anpltidue node data

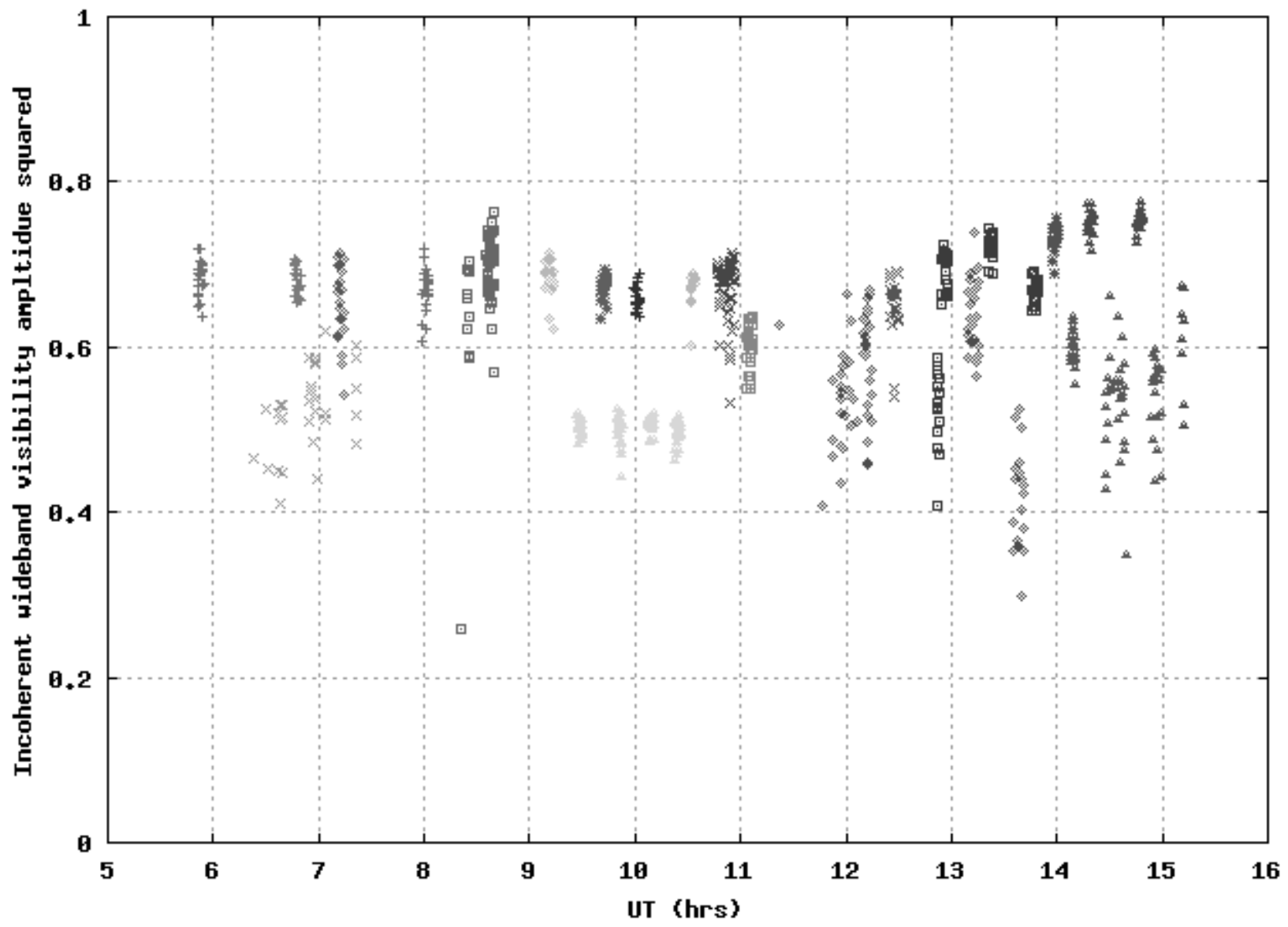

Figure 1: An example night of KI data showing the incoherent visibility amplitude squared as a function of time. Different sources are plotted with different symbols. On this night, several different science sources were observed and as can be seen, some of the science targets were partially resolved. For example, the object at V2 0.5 between 9 and 11 UT hours is a binary system. Data plotted here are averaged visibilities from the standard processing pipeline and each point is a 5 second average. 


\subsection{Visibility amplitude science}

To date, visibility amplitude observations with the Keck Interferometer have resulted in two refereed science publications ${ }^{[8,9]}$. The NGC 4151 result is discussed elsewhere in this volume ${ }^{10}$. An array of astrophysical topics has been pursued by the shared-risk teams, and the astronomical sources observed so far include:

- Young stellar objects: T Tauri stars (circumstellar disk size scales), Herbig Ae/Be stars (circumstellar disk size scales), FU Ori objects (circumstellar disk size scales) and binaries (mass determination).

- Wolf-Rayet stars (circumstellar material).

- Main sequence stars (stellar diameters) and binaries (mass determination).

- Active galactic nuclei (nuclear emission size scales), see Ref. 10.

This list demonstrates the wide range of astrophysical topics for which near-infrared interferometry is a relevant and exciting new observational technique.

\subsection{Studies of Young Stellar Objects}

In this section, observations of young stellar objects are discussed in detail as an example of the science currently being pursued at KI. T Tauri stars are pre-main-sequence objects with stellar masses less than 2 solar masses, and ages of 1 to 10 million years, while Herbig Ae/Be stars are their more massive counterparts. The canonical model for low mass star formation comprises a central star surrounded by a circumstellar disk of size $\sim 100 \mathrm{AU}$, a collimated jet or outflow, and perhaps some residual material in a more extended envelope. Due to the high resolution required (1 AU subtends 7 milliarcseconds at the distance to the nearest star forming regions), direct observations of the central region have been limited to date. Characterizing the inner disk properties is important for understanding hydrodynamic disk winds and the initial conditions of planet formation. Given the spatial resolution of current facilities, long-baseline infrared interferometry provides an ideal method for observing these inner regions, which are traced by material thermally emitting in the near-infrared. Previous interferometric observations have resolved several types of young stellar objects: FU Ori ${ }^{11}, \mathrm{~T}$ Tauri ${ }^{12}$, and Herbig AeBe ${ }^{13}$ sources. For the $\mathrm{T}$ Tauri and Herbig objects, the measured visibilities are generally not consistent with a geometrically flat disk with an inner radius of roughly a few stellar radii as predicted by spectral energy distribution fitting. Refs. 14 and 15 proposed that the inner radius is located at the destruction radius for directly heated dust, which can resolve this discrepancy. A recent addition to this theory includes the accretion luminosity as well as the stellar luminosity in the determination of the dust destruction radius ${ }^{16}$.

Observations of young stellar objects with KI have been pursued by the Monnier and Danchi shared-risk teams and by scientists on the development team. Here I will present preliminary results on T Tauri and Herbig Ae/Be stars from the internal and Monnier teams. One of the goals of these studies is to observe sources with a range of spectral types and disk indicators, including infrared excess and $\mathrm{H}$-alpha emission lines. A simple ring geometry was used to compare the observations to the model discussed above, where the inner ring radius is located at the dust destruction temperature. The measured visibilities were fit with a model comprising the central star, which is unresolved for these sources, and a ring component, representing the inner disk radius. None of the sources have sufficient hour angle coverage to significantly constrain the inclination and position angles of the emission source, so a face-on geometry was used. Analysis of the Herbig sources was taken from Ref. 16. For the T Tauri stars, the stellar contribution and luminosities were taken from the literature ${ }^{[16,18]}$. The dust sublimation radius was calculated using the stellar and total luminosities following Ref. 19, where a dust grain size of 0.3 microns and a dust sublimation temperature of $1500 \mathrm{~K}$ were used. The results for stars with spectral types ranging from M0 to B8 are plotted in Figure 2, which plots the calculated dust sublimation radius against the measured ring size. These two quantities are equal along the diagonal line. The $\mathrm{T}$ Tauri sources (lower left corner) have measured radii larger than predicted. Estimates of the total (stellar + accretion) luminosity were available for four of the sources and those points are given by the squares. However, even using the larger total luminosity does not eliminate the discrepancy between the predicted and measured radii. The source with a 0 AU measured radius is a Herbig Be star with little or no infrared excess which was unresolved in the KI observations. 


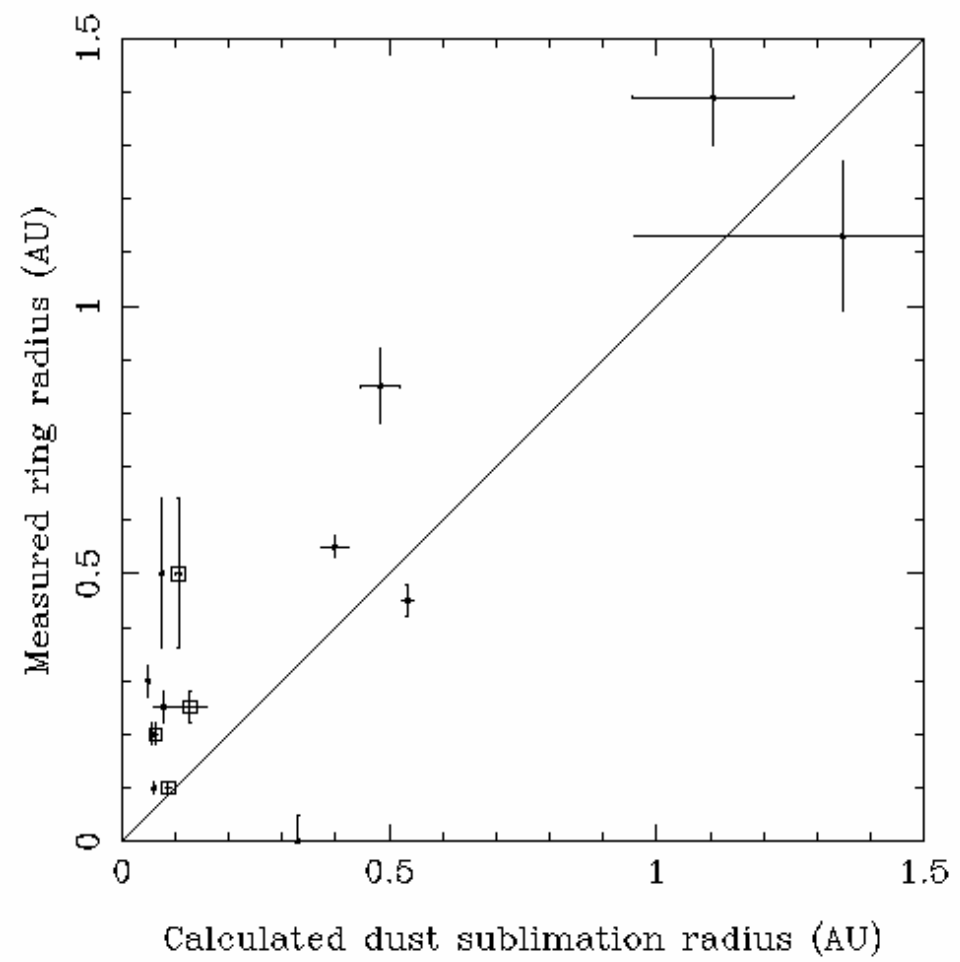

Figure 2: The measured ring radius plotted against the calculated dust sublimation radius for a group of Herbig Ae/Be and T Tauri stars. The dust sublimation temperature was assumed to be $1500 \mathrm{~K}$ and a grain size of 0.3 microns was used. The calculated dust sublimation radius is shown for the stellar luminosity for all sources (dots) and the total luminosity (squares) where available.

\section{Nulling science}

One of the goals of NASA's Origins program is to find Earth-like planets around other stars. A set of projects, including the Keck Interferometer, the Space Interferometry Mission, and the Large Binocular Telescope Interferometer lead up to the Terrestrial Planet Finder, which will have the capability to observe and characterize Earth-like planets. The role of the Keck-Keck Interferometer in this search is to characterize the environment of nearby potential TPF target stars. The solar system contains dust within the plane of the planetary orbits called zodiacal dust and other planetary systems are expected to have the same material. Although this dust may not have a large mass, the large surface area means that the emission can be significant in obscuring the emission from a planet located in this dust cloud. The nulling interferometry mode of KI will characterize the level of exo-zodiacal dust around nearby stars. The nulling mode is necessary to block to light from the central star in order to see emission from the dust which is orders of magnitude fainter. The goal of this mode is to be able to detect exo-zodiacal clouds with 30 times the dust level of our own solar system. 
In addition to the Key Science of exo-zodiacal dust measurements, the nulling mode will be useful for a variety of science observations which require blocking the central star to observe fainter nearby sources of emission. For instance, once a young star has accreted and dispersed the majority of the circumstellar disk and envelope from which it formed, the central star is much brighter than the remaining disk material. Nulling interferometry observations of these objects, and older main sequence stars with debris disks, such as the so-called Vega-like systems can be used to characterize the disk. This technique can also be used at the other end of the stellar lifecycle for studying the mass loss in evolved stars.

\section{Differential phase science}

As of May, 2004, high precision radial velocity observations have discovered 110 planets around other stars (see http://exoplanets.org). Although the radial velocity technique provides only a lower limit to the mass, the canonical model of these systems is as planets, rather than brown dwarfs or very low mass stars. While the radial velocity studies are excellent for discovering these systems, they provide no information on the physical properties of the exo-planets. In a very small number of these systems, the planet transits the central star, allowing the planet size and mass to be measured, and the density to be calculated. But for most systems, the general composition is unknown.

Of the currently known systems, 17 have orbital radii less than $0.1 \mathrm{AU}\left(1 \mathrm{AU}=1.5 \times 10^{8} \mathrm{~km}\right)$. This collection of exoplanets is often called Hot Jupiters as their small orbital radii will result in high surface temperatures. A high surface temperature (>1000 Kelvin) will result in a flux ratio of roughly $10^{4}$ in the near-infrared for planets around solar-like stars and the flux ratio will vary with wavelength. The differential phase technique will use the wavelength dependence of this flux ratio to directly detect the Hot Jupiter. Although the absolute phase difference is too small for KI to detect, the differential phase technique will measure the relative shift between wavelengths with sufficient precision to detect Hot Jupiters. The differential phase signature measured at different orbital phases will allow determination of the true mass and spectral characterization. There is a wide range of predictions on the resulting atmospheric composition for Hot Jupiters.

The differential phase technique can also be applied to other astronomical systems where a color difference across the near-infrared exists between the system components. For example, the flux ratios of a low mass/high mass stellar binary can be comparable to those in the Hot Jupiter observing. Unlike high precision radial velocities observations, which rely on hundreds of spectral lines to produce the necessary velocity precision and thus are constrained to specific spectral types, differential phase can be used for any stellar spectral type.

\section{Acknowldegements}

The Keck Interferometer is funded by the National Aeronautics and Space Administration (NASA). Part of this work was performed at the Jet Propulsion Laboratory, California Institute of Technology, and at the Michelson Science Center (MSC), under contract with NASA. Observations were obtained at the W. M. Keck Observatory, operated as a scientific partnership among California Institute of Technology, the University of California, and NASA. The Observatory was made possible by the generous financial support of the W. M. Keck Foundation. The authors wish to recognize and acknowledge the very significant cultural role and reverence that the summit of Mauna Kea has always had within the indigenous Hawaiian community. We are most fortunate to have the opportunity to conduct observations from this mountain. The author would like to thank R. Millan-Gabet for work on the T Tauri data. This work has used software from the MSC; the SIMBAD database, operated at CDS, Strasbourg, France; and the NASA/IPAC Infrared Science Archive, operated by JPL under contract with NASA.

\section{References}

1. Colavita, M., Wizinowich, P., and Akeson, R., "Keck Interferometer status and plans", SPIE, this volume.

2. Wizinowich, P.L., Le Mignant, D., Stomski, P.J., Acton, D.S., Contos, A.R., and Neyman, C.R., "Adaptive optics developments at Keck observatory", SPIE proceedings, vol. 4839, p. 9. 
3. Vasisht, G., Booth, A.J., Colavita, M.M., Johnson, R.L., Ligon, E.R., Moore, J.D., and, D.L., "Performance and verification of the Keck interferometer fringe detection and tracking system”, SPIE proceedings, vol. 4838 , p. 824.

4. Colavita, M.M., "Fringe Visibility Estimators for the Palomar Testbed Interferometer", Publications of the Astronomical Society of the Pacific, Vol. 111, p. 111.

5. Serabyn, E., "The Keck interferometer nuller", SPIE, this volume.

6. Vasisht, G., "Differential phase interferometry with the Keck Telescopes", SPIE, this volume.

7. Boden, A.F., Colavita, M.M., van Belle, G.T., and Shao, M.," Visibility calibrations with the Palomar Testbed Interferometer", SPIE proceedings, vol. 3350, p. 872

8. Colavita M., et al, “Observations of DG Tauri with the Keck Interferometer", Astrophysical Journal Letters, vol. 592, p. 83.

9. Swain, M., et al, "Interferometer Observations of Subparsec-Scale Infrared Emission in the Nucleus of NGC 4151", Astrophysical Journal Letters, vol. 596, p. 163.

10. Swain, M., "Observing NGC 4151 with the Keck interferometer", SPIE, this volume.

11. Malbet, F. et al., "FU Orionis Resolved by Infrared Long-Baseline Interferometry at a 2 AU Scale", Astrophysical Journal, vol. 507, p. 149.

12. Akeson, R.L., Ciardi, D.R., van Belle, G.T., Creech-Eakman, M.J., and Lada, E.A.," Infrared Interferometric Observations of Young Stellar Objects", Astrophysical Journal, vol. 543, p. 313.

13. Millan-Gabet, R., Schloerb, F.P., and Traub, W.A.," Spatially Resolved Circumstellar Structure of Herbig AE/BE Stars in the Near-Infrared", Astrophysical Journal, vol. 546, p. 358.

14. Tuthill, P.G., Monnier, J.D., and Danchi, W.C.," A dusty torus around the luminous young star LkHalpha 101", Nature, vol. 409, p. 1012.

15. Natta, A., Prusti, T., Neri, R., Wooden, D., Grinin, V.P., and Mannings, V., "A reconsideration of disk properties in Herbig Ae stars", Astronomy and Asrophysics, vol. 371, p. 186.

16. Muzerolle, J., Calvet, N., Hartmann, L., and D'Alessio, P.," Unveiling the Inner Disk Structure of T Tauri Stars”, Astrophysical Journal Letters, vol. 597, p. 149.

17. Monnier, J.D., et al.," Young Stellar Objects with the Keck Interferometer", American Astronomical Society Meeting, 203.

18. Kenyon, S.J. and Hartmann, L.," Pre-Main-Sequence Evolution in the Taurus-Auriga Molecular Cloud", Astrophysical Journal Supplemental Series, vol. 101, p. 117.

19. Monnier, J.D. and Millan-Gabet, R.," On the Interferometric Sizes of Young Stellar Objects", Astrophysical Journal, vol. 579, p. 694. 